\title{
Selective lentiviral-mediated suppression of microRNA124a in the hippocampus evokes antidepressants-like effects in rats
}

\author{
Amine Bahi $^{a, *}$, Vijay Chandrasekar ${ }^{b}$, Jean-Luc Dreyer ${ }^{c}$ \\ ${ }^{a}$ Department of Anatomy, Tawam Medical Campus, CMHS, United Arab Emirates University, Al Ain, \\ United Arab Emirates \\ ${ }^{\mathrm{b}}$ Institute of Neuropathology, University Hospital of Zurich, Schmelzbergstrasse 12, CH-8091 Zurich, Switzerland \\ ${ }^{\mathrm{C}}$ Division of Biochemistry, Department of Medicine, University of Fribourg, $\mathrm{CH}-1700$ Fribourg, Switzerland
}

\author{
KEYWORDS \\ BDNF; \\ Social defeat stress; \\ Depression; \\ Lentiviral vector; \\ microRNAs; \\ miR124a
}

\begin{abstract}
Summary Several lines of evidences suggest that the brain-derived neutrophic factor (BDNF) is implicated in the pathophysiology of depression. However, the molecular mechanisms are not fully understood. In the current study we aimed to investigate how genetic modulation of BDNF in the hippocampus using microRNa124a (miR124a)-expressing lentiviral vectors (LV) might affect depression-like behavior in adult rats. For this purpose, we assessed the expression level of miR124a and its direct target BDNF in the hippocampus and the cortex after 21-days exposure to social defeat stress. Results demonstrated that miR124a was up-regulated in the hippocampus but not in the cortex. In contrast, and as expected, BDNF transcripts were down-regulated. In a different set of experiments, male Wistar rats received bilateral intra-hippocampal or intracortical infusions of BDNF- and miR124a-expressing lentiviral vectors and depression-like behavior was assessed after 21-days social defeat stress using the novelty suppressed feeding, the sucrose preference and the forced swim tests. The results indicated that miR124a overexpression exacerbated depression-like behavior. However, an anti-depressant like effect was observed when BDNF or miR124a-silencers (siR124a) were injected into the hippocampus. Importantly, when expressed into the cortex, LV-miR124a, LV-siR124a and LV-BDNF had no effect on depression. Our findings indicate that hippocampal miR124a and its direct target BDNF play an important role in depression-like behavior. Taken together, the current results reveal, for the first time, a potential molecular regulation of miR124a on BDNF, and the pronounced behavioral consequences of this regulation shed light on the mechanisms underlying BDNF anti-depressant potential.

(C) 2014 Elsevier Ltd. All rights reserved.
\end{abstract}

Abbreviations: BDNF, brain-derived neurotrophic factor; FST, forced swim test; Hipp, hippocampus; LV, lentiviral vector; miRNA, microRNA; NSFT, novelty suppressed feeding test; SPT, sucrose preference test.

* Corresponding author at: Department of Anatomy, College of Medicine \& Health Sciences, United Arab Emirates University, PO Box 17666, Al Ain, United Arab Emirates. Tel.: +971 37137 516; fax: +971 37672033.

E-mail address: amine.bahi@uaeu.ac.ae (A. Bahi). 


\section{Introduction}

Depression is a prevalent, highly debilitating mental disorder affecting up to $15 \%$ of the population at least once in their lifetime, with huge costs for society. Neurobiological mechanisms of depression are still not well known, although there is consensus about interplay between genetic and environmental factors (Masi and Brovedani, 2011). Antidepressant medications are frequently used in depression, but at least $50 \%$ of patients are poor responders, even to more recently discovered medications. There is a clear evidence for altered endocrine factors and metabolic dysregulation in mood disorders (Hendrickx et al., 2005). However, clinical response only occurs following weeks to months of treatment and only chronic treatment is effective (Masi and Brovedani, 2011), suggesting that actions beyond the rapidly occurring effect of enhancing monoaminergic systems, such as adaptation of these systems, are responsible for the effects of antidepressants.

Recent studies have shown that certain aspects of depression result from maladaptive stress-induced neuroplastic changes in specific neural circuits of the CNS (Nestler et al., 2002). These studies emphasize that neuronal plasticity plays an important role in the recovery from depression and they indicate that an impairment of synaptic plasticity, particularly in the hippocampus, may be a core factor in the pathophysiology of depression. The abnormal neural plasticity may be related to alterations in the levels of neurotrophic factors, namely brain-derived neurotrophic factor (BDNF), which play a central role in plasticity. As BDNF is repressed by stress, epigenetic regulation of the BDNF gene may play an important role in depression (Masi and Brovedani, 2011). In turn as a treatment, antidepressant drugs and electroconvulsive shock treatment increase the expression of BDNF and its receptor TrkB (Castren and Rantamaki, 2010).

Neurotrophins constitute a family of proteins that stimulate and control neurogenesis during development and BDNF is among the most active (Zigova et al., 1998). BDNF plays a key role on the central and peripheral nervous system to support the survival of existing neurons, and to induce neurogenesis and stimulate synaptic plasticity and synaptogenesis (Acheson et al., 1995). It is mainly active in brain areas vital to learning, memory, and higher thinking, including the hippocampus, the cortex, and basal forebrain (Yamada and Nabeshima, 2003). In addition, BDNF regulates synaptic plasticity in neuronal networks involved in several brain disorders including bipolar disorder, as well as schizophrenia, obsessive-compulsive disorder, Alzheimer's disease, Huntington's disease, Rett syndrome, and dementia (Schinder and Poo, 2000). Furthermore, stress exposure decreases the expression of BDNF, and, if exposure is persistent, this leads to an eventual atrophy of the hippocampus, which also takes place in humans suffering from chronic depression. In turn, regulation of BDNF may reverse stress-induced deficits in structural and synaptic plasticity in the brain, yielding increased ability to cope with environmental challenges that may precipitate or exacerbate depressive episodes (Castren and Rantamaki, 2010). Low BDNF and depression are most probably causally related, since antidepressants indeed increase BDNF signaling and synthesis in the hippocampus (Schmidt and Duman, 2010). Although it is now firmly established that BDNF signaling plays an important role in the mechanism of action of antidepressant drugs, yet the role of BDNF in the pathophysiology of depression is less clear (Castren and Rantamaki, 2010). Furthermore, there is strong evidence that peripheral growth factors, including BDNF, pro-inflammatory cytokines, endocrine factors, and metabolic markers contribute to the pathophysiology of major depressive disorder and antidepressant response (Schmidt et al., 2011). As a matter of fact BDNF levels are decreased in the blood of depressed patients and can be normalized with successful antidepressant treatment (Sen et al., 2008), although the origin and role of serum BDNF is unclear (Karege et al., 2005). Chronic peripheral administration of BDNF produces antidepressant and anxiolytic behavioral responses in animal models, increases the survival rate of newborn neurons, and increases BDNFmediated signaling in the adult hippocampus (Schmidt and Duman, 2010), an evidence that peripheral BDNF has functional actions within the brain and on behavior, and that serum BDNF may be a relevant biomarker for depression and treatment response.

Neurotrophin BDNF acts via its receptor Tropomyosinrelated kinase $B$ receptor (TrkB or $\mathrm{p} 75$ ), promoting intracellular signaling that is yielding to protein synthesis (Soppet et al., 1991). The induction of protein synthesis by BDNF critically contributes to enduring modifications of synaptic function, but how BDNF selectively affects only a minority of expressed mRNAs is poorly understood. It has been established that BDNF rapidly elevates Dicer, increasing mature microRNAs (miRNA) levels and inducing RNA processing bodies in neurons (Huang et al., 2012).

MiRNAs are small non-coding RNAs of about 22-nucleotides in length regulating gene expression at post-transcriptional level that act as key regulators of all cellular pathways (Treiber et al., 2012). After transcription, the mature miRNA is incorporated into a miRNA-protein complex, where it directly interacts with a member of the Argonaute protein family. The miRNA guides such protein complexes to partial complementary target sites, which are typically located in the $3^{\prime}$ untranslated region (UTR) of mRNAs leading to inhibition of gene expression. MiRNA activity and abundance is regulated on various levels ranging from transcription and processing to target site binding and miRNA stability (Treiber et al., 2012). A single miRNA may specifically regulate gene expression of over hundred different targets. Among them, miR124a is strongly expressed in the brain; miR124a regulates adult neurogenesis (Cao et al., 2007), and promotes neuronal differentiation (Makeyev et al., 2007) and it is involved in several brain disorders (Qi et al., 2012). In addition, miR124a also strongly affects the expression of several neurotransmitters, including mainly BDNF (Bahi and Dreyer, 2013; Chandrasekar and Dreyer, 2009, 2011). This prompted us to investigate the potential role of miR124a in the ethiology of depression.

For this purpose, in this study we assessed the expression levels of miR124a and its direct target BDNF in the rat brain following exposure to 21 -days social defeat stress. In addition, our major goal in the present studies was to examine the effects of lentiviral-mediated genetic modulation of BDNF and miR124a in male Wistar rats on performance in three behavioral tests following exposure to chronic social defeat stress. 


\section{Materials and methods}

\subsection{Animals}

Experimental male Wistar rats $(\sim 200 \mathrm{~g})$ were single-housed in standard plexiglas cages at a room temperature of approx. $22{ }^{\circ} \mathrm{C}$ with a $12 \mathrm{~h} / 12 \mathrm{~h}$ light/dark cycle and allowed to adapt to this environment for a period of 7 days before the experiments began. All rats were bred in the local central animal facility of the CMHS and were kept under standard laboratory conditions. Bedding was produced locally and autoclaved before use and rats had free access to tap water and standard rodents chow diet obtained from the National Feed and Flour Production and Marketing Company LLC (Abu Dhabi, UAE). All the experimental procedures were approved by the institutional Research Ethics Committee (Protocol No A17-12).

\subsection{Tissue collection and quantification of BDNF and miR124a after social defeat stress}

Brains from decapitated control and stressed rats were rapidly removed and brain regions (whole cerebral cortex and hippocampus) were dissected out on ice. Tissue samples were immediately frozen in TRizol and stored at $-80^{\circ} \mathrm{C}$ until analysis. The micro-dissection procedure was performed according to rat stereotaxic coordinates (Paxinos and Watson, 1998).

Reactions were essentially carried out as described previously (Bahi and Dreyer, 2013; Chandrasekar and Dreyer, 2009). In brief, total RNA from brain regions was isolated using the TRizol according to the manufacturer instructions. Total RNA (5 $\mu \mathrm{g}$ per sample) was reverse transcribed using Oligo-dT standard primers. For qPCR, $20 \mu \mathrm{l}$ of amplification mixture with SYBRGreen Kit was used containing $3 \mu \mathrm{l}$ of CDNA and $100 \mathrm{nM}$ primers. Primer sequences used were: GAPDH: 5'-ATG ACT CTA CCC ACG GCA AG-3' and 5'-CAT ACT CAG CAC CAG CAT CAC-3'; miR124a: 5'-TCC GTG TTC ACA GCG GAC-3' and 5'-CAT TCA CCG CGT GCC TTA-3' and BDNF: $5^{\prime}$-GGT TCG AGA GGT CTG ACG AC-3' and 5'-CAA AGG CAC TTG ACT GCT GA-3' (Bahi et al., 2008a; Bahi and Dreyer, 2013). The PCR protocol used consisted of a 45 -s denaturation at $94{ }^{\circ} \mathrm{C}$ followed by $45-\mathrm{s}$ annealing and extension at $64^{\circ} \mathrm{C}$ for 40 cycles.

\subsection{Lentiviral constructions and production}

Our lentivirus derived plasmid (pTK431), driven by the cytomegalovirus (CMV) promoter, carried the transgenes for BDNF, miR124a or siR124a described in our previous studies (Bahi et al., 2008a; Bahi and Dreyer, 2013; Chandrasekar and Dreyer, 2009). The empty vector (LV-Mock) was used as negative control. All constructions were confirmed by sequencing. Viral vector generation was obtained by cotransfection of HEK293T cells by the calcium phosphate method on $10-\mathrm{cm}$ plates with $20 \mu \mathrm{g}$ of PTK431, $15 \mu \mathrm{g}$ of $\mathrm{p} \Delta \mathrm{NRF}$, and $5 \mu \mathrm{g}$ of pMDG-VSV-G. The supernatant was harvested 24 and $72 \mathrm{~h}$ after infection and viral particles were purified by ultracentrifugation (Bahi et al., 2005, 2008b).

\subsection{Stereotaxic injection of LV-BDNF, LV-Mock, LV-miR124a and LV-siR124a}

For stereotaxic surgery, rats were anesthetized with a ketamine/xylazine mixture $(100 \mathrm{mg} / \mathrm{kg}$ and $10 \mathrm{mg} / \mathrm{kg}$ respectively, i.p.) and installed in a stereotaxic frame. Using a precision Hamilton micro-syringe with a $26 \mathrm{G}$ needle, rats were bilaterally infused with $1 \mu \mathrm{l}$ viral solution using the following coordinates: hippocampus [1st injection: $4.8 \mathrm{~mm}$ posterior to Bregma, $\pm 2.5 \mathrm{~mm}$ lateral the medial suture, $3.5 \mathrm{~mm}$ ventral to the skull surface. 2nd injection: $4.8 \mathrm{~mm}$ posterior to Bregma, $\pm 5 \mathrm{~mm}$ lateral the medial suture, $6 \mathrm{~mm}$ ventral to the skull surface]. Cortex [1st injection: $2.2 \mathrm{~mm}$ anterior to Bregma, $\pm 3 \mathrm{~mm}$ lateral the medial suture, $2 \mathrm{~mm}$ ventral to the skull surface. 2nd injection: $2.2 \mathrm{~mm}$ anterior to Bregma, $\pm 4.5 \mathrm{~mm}$ lateral the medial suture, $4 \mathrm{~mm}$ ventral to the skull surface]. All viral-injections were performed according to the rat stereotaxic coordinates (Paxinos and Watson, 1998). Rats were left to recover for 7 days before being exposed to the social defeat stress procedure. After recovery and in the 1 st set of experiments, we ended up with 19 rats that were injected into the hippocampus (LV-Mock $n=9$, LV-BDNF $n=10$ ) and 16 rats that were injected into the cortex (LV-Mock $n=8$, LV-BDNF $n=8$ ). In the 2 nd set of experiment we ended up with 24 rats that were injected into the hippocampus (LV-Mock $n=7$, LV-miR124a $n=9$, siR124a $n=8)$ and 20 rats that were injected into the cortex (LV-Mock $n=6$, LV-miR124a $n=7$, siR124a $n=7$ ).

\subsection{Social defeat stress}

The social defeat stress model of depression was based on the resident-intruder paradigm (Korte et al., 1990). In brief, experimental-rats were exposed to a large dominant adult ( 400 g) male Wistar aggressor rat for 30 min each day over a total of 21 days. After contact, the experimental-rat was separated from the aggressor resident-rat using a cage that was divided into two parts by a perforated divider. Thus, the experimental-rat and the resident-rat were maintained in visual and sensory contact for the next $24 \mathrm{~h}$ without physical contact. Every 4th day the experimental-rat was exposed to a new aggressor. Resident aggressor rats were selected for their level of aggressiveness by counting the latency to attack a naive Wistar rat (maximum 2 min delay). To promote their aggressive behavior, the resident-rats were housed with females 10 days prior to the social defeat stress test to ensure that they accept and defend their cage as home cage (territory).

\subsection{Depression-like behavioral tests}

\subsubsection{Novelty suppressed feeding (NSF) test}

The novelty suppressed feeding procedure was performed as described previously (Bahi and Dreyer, 2012). In brief, $24 \mathrm{~h}$ prior to testing, rats were deprived of food but not water. On the test day, each rat was introduced into a white plastic open arena $(32 \mathrm{~cm} \times 32 \mathrm{~cm})$ covered with wood-chip bedding, and on which center several pellets of food were put on a circular paper. The latency to bite the food pellet was 
measured as the test main parameter. Immediately after the animal commenced eating, the rat was immediately removed from the testing box and returned to its home cage with access to food. Therefore, the total pellet consumption was measured for a further 90 min period in order to evaluate home cage food intake.

\subsubsection{Sucrose preference test (SPT)}

For the SPT, a two-bottle choice procedure was used to test for differences between the groups for their relative preference for sucrose over water. The rats were given free choice between $2 \%$ sucrose-solution and tap water for $24 \mathrm{~h}$. One week before starting, experimental-rats had been habituated to two-bottle choice conditions and the position of the bottles was switched after every $24 \mathrm{~h}$ to prevent any side preference in drinking behavior. No previous food or water deprivation was applied before the test. Preference was measured as follows: (i) sucrose consumption (ml); (ii) water consumption $(\mathrm{ml})$; and (iii) total liquid (sucrose + water). At the end of the test, the bottles were removed and the consumption was noted. The preference for sucrose was calculated as percentage of consumed sucrose-solution to the total amount of liquid drunk (Hennebelle et al., 2012; Zurita et al., 1996).

\subsubsection{Forced swim test (FST)}

The FST was performed according to the method originally reported by Porsolt and colleagues (Porsolt et al., 1977) and this procedure was successfully used in the laboratory in our previous studies (Bahi and Dreyer, 2012). In brief, glass beakers were filled with tap water $\left(22-26^{\circ} \mathrm{C}\right)$. Rats were placed into the test beaker and were unable to escape or rest by touching the bottom of the beaker. The cylinders were emptied and cleaned between rats. Sessions lasted 6 min and the following behavioral parameters were measured: immobility, swimming and climbing. Immobility was defined as the absence of all motions except those required for keeping the rat's head above water. The swimming behavior was considered if a rat was actively making swimming movements that caused it to move within the center of the cylinder. The climbing behavior was recorded if a rat was making forceful thrashing movements with its forelimbs against the walls of cylinder. The data are expressed as the mean time $( \pm$ SEM) of immobility, swimming and climbing within the 360 -s observation period.
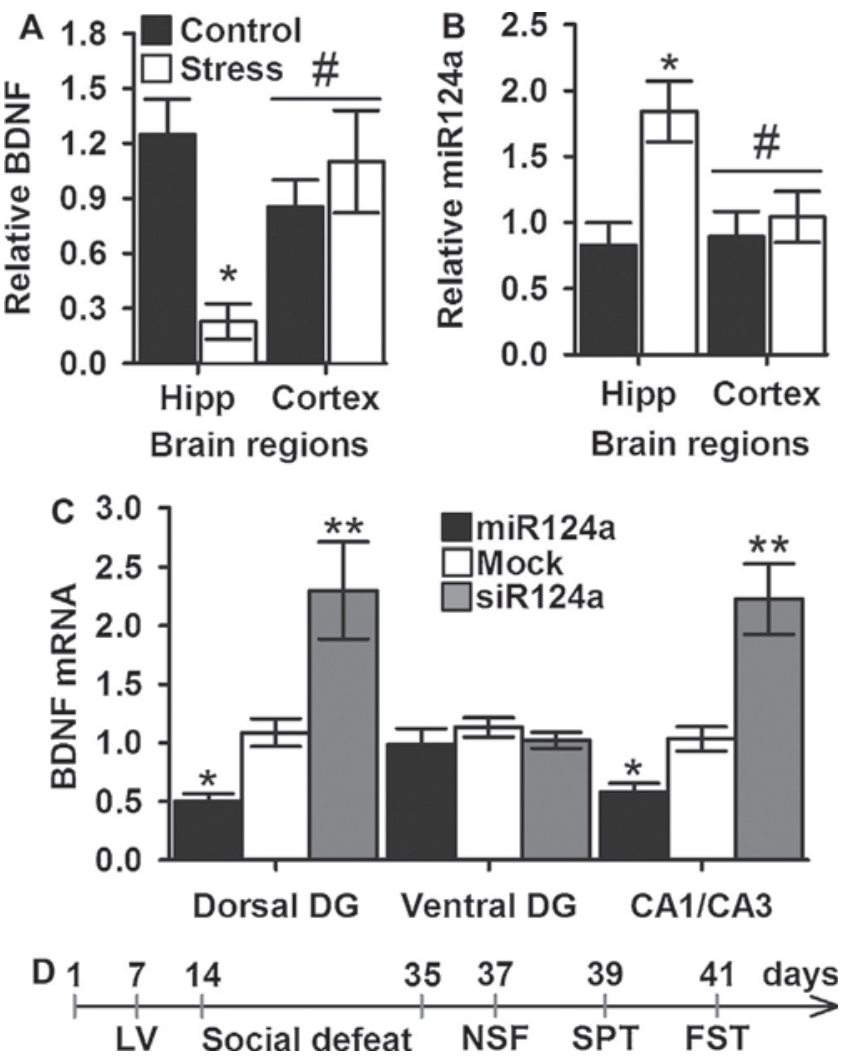

Figure 1 Effect of chronic social defeat on (A) BDNF and (B) miR124a expression in the rat hippocampus and cortex. Expression levels were normalized against GAPDH in the corresponding samples. Histograms represent mean $\pm \mathrm{SEM}, n=6$. ${ }^{*}$ Significant differences between control and stress $(p<0.05)$. "Significant differences between the hippocampus and the cortex $(p<0.05)$ (two-way ANOVA, Bonferroni's post hoc test), (C) effect of lentiviral injection on BDNF mRNA expression in the dorsal DG, ventral DG and the CA1/CA3. Expression levels were normalized against GAPDH in the corresponding samples. Histograms represent mean $\pm \mathrm{SEM}, n=7$. ${ }^{\circ}$ Significant differences between LV-Mock and LV-miR124a $(p<0.05) .{ }^{* *}$ Significant differences between LV-Mock and LV-siR124a $(p<0.001)$ (twoway ANOVA, Bonferroni's post hoc test) and (D) schematic representation of the experimental procedures. The timeline shows sequence and duration of experimental protocols of the effect of viral injection on chronic social defeat-induced depression-like behavior. 


\subsection{Statistical analysis}

For statistical comparisons, the software package SPSS (version 20.0) was used. All data were expressed as means \pm SEM. BDNF and miR124a mRNA expression was analyzed using a two-way analysis of variance (ANOVA) with treatment (control vs. stress) and region (hippocampus vs. cortex) as the between subject factors. Data obtained from the behavioral tests were analyzed using a two-way ANOVA with virus and region as the between subject factors. Post hoc individual mean comparisons were performed with the Bonferroni's test when $F$ values were significant. The level of statistical significance was set at $p<0.05$ at all times.

\section{Results}

\subsection{Effects of social defeat stress on brain expression of BDNF and miR124a}

Fig. 1 shows the effects of chronic social defeat stress on the expression of BDNF in the hippocampus and the cortex detected by RT-PCR. As shown in Fig. 1A, 21-days social defeat stress caused a significant down regulation ( $\sim 4$ folds) of BDNF mRNA in the hippocampus but not in the cortex. A two-way ANOVA revealed a main effect of stress $\left(F_{(1,20)}=4.027\right.$, $p=0.058)$; but the main effect of brain region was not significant $\left(F_{(1,20)}=1.533, p=0.230\right)$. Nevertheless, the interaction between stress and brain region was significant $\left(F_{(1,20)}=10.926, p=0.004\right)$. These findings demonstrated that 3 weeks of social defeat stress in rats down-regulated hippocampal, but not cortical, BDNF mRNA expression.

Fig. 1B shows the miR124a expression levels in the investigated brain regions hippocampus and cortex. All data are means \pm SEM of the particular brain specimens from the control $(n=6)$ and socially defeated $(n=6)$ groups of rats. Results show that miR124a is expressed in each of the investigated brain regions, although at different levels. In fact, social defeat stress produced a significant increase ( $\sim 2$ folds) of miR124a in the hippocampus but not in the cortex. Two-way ANOVA indicated that the individual [stress: $\left(F_{(1,20)}=8.689\right.$, $p=0.008)$; brain region $\left(F_{(1,20)}=3.446, p=0.078\right)$ ] and interactive $\left(F_{(1,20)}=4.784, p=0.041\right)$ effects of stress and brain regions were statistically significant. These results indicated that 3-weeks of social defeat stress in rats increased miR124a expression into the hippocampus, but not into the cortex.

In order to provide more convincing evidence that BDNF was directly regulated by miR124A, rats were stereotaxically injected with LV-Mock, LV-miR124a or LV-siR124a in the hippocampus and BDNF mRNA was quantified using RT-PCR. The regions chosen to quantify BDNF expression were the dorsal and ventral dentate gyrus (DG) and the CA1/CA3 region. As depicted in Fig. 1C, two-way ANOVA analysis with virus and sub-region as the between subject factors revealed a significant effect of viral injection $\left(F_{(2,54)}=28.992, p<0.0001\right)$ but no effect of sub-region was detected $\left(F_{(2,54)}=1.653\right.$, $p=0.205)$. Nevertheless, the interaction between the two factors was significant $\left(F_{(4,54)}=7.297, p<0.001\right)$. Post hoc evaluations revealed that LV-miR124a and LV-siR124a only affected BDNF expression in the dorsal DG as well as in the CA1/CA3 sub-regions but not in the ventral DG.
Based on these findings we decided to investigate the potential protective role of BDNF and miR124a-silencers overexpression into the hippocampus on social defeat stress-induced depression-like behavior in rats. The experimental timeline is depicted in Fig. 1D.

\subsection{Effects of BDNF overexpression on social defeat stress-induced depression}

The novelty-induced suppression of feeding (NSF) test assesses depression- and anxiety-like phenotypes by measuring the latency of rats to eat food in a novel anxiogenic open field following food deprivation (Bodnoff et al., 1989). Rats were stereotaxically injected into the hippocampus or into the cortex and exposed to the social defeat stress 7 days later as described in Section 2. We should emphasize that one rat from each group was removed from the analysis as they escaped from the arena. Therefore the group sizes were: hippocampus (LV-Mock $n=8$, LV-BDNF $n=10$ ); cortex (LVMock $n=8$, LV-BDNF $n=7$ ). Using a two-way ANOVA test we found a significant effect of virus for the latency to bite a food pellet at the center of the arena, with the hippocampus BDNF-injected group having a significantly shorter latency to feed [main effect of virus: $\left(F_{(1,29)}=13.704\right.$, $p=0.001)$; main effect of brain region: $\left(F_{(1,29)}=11.558\right.$, $p=0.002)]$. More importantly, the interaction between the two factors was significant $\left(F_{(1,29)}=9.918, p=0.004\right)$ (Fig. 2A). In addition and as depicted in Fig. 2B, BDNF rats did not differ from Mock-injected rats in their food intake in the home cage during a 90 -min period immediately following the latency test [main effect of virus: $\left(F_{(1,29)}=0.082\right.$, $p=0.776)$; main effect of brain region: $\left(F_{(1,29)}=0.048\right.$, $p=0.829)$; interaction between virus and brain region: $\left.\left(F_{(1,29)}=0.254, p=0.618\right)\right]$. Finally, both Mock- and BDNFoverexpressing groups did not differ in the amount of weight lost during the $24 \mathrm{~h}$ food deprivation period (data not shown). These findings strongly suggest that reduced latency to feed was directly related to lower anxiety levels in the hippocampus, but not the cortex, of BDNF-overexpressing rats.

In order provide an appreciation of the precise magnitude of the stressors alone on the various behavioral tests and how the various lentiviral treatments alone affected these tests, we performed a further experiment using non-stressed control group that is given the various lentiviral treatments in the hippocampus (not the cortex) and then subsequently tested (Supplementary Fig. 1). It should be emphasized that the data with non-stress controls and stress animals are a combination of separate experiments, which is far from the optimal design.

Rats were then assessed in the sucrose preference test (SPT). This is a model of anhedonia, a core symptom of depression, in which rats fail to elicit pleasure from an activity that is normally enjoyable (Sofia and Knobloch, 1976). The groups of rats used were as follow: hippocampus (LV-Mock $n=9$, LV-BDNF $n=10$ ); cortex (LV-Mock $n=8$, LVBDNF $n=8$ ). The SPT scores are shown in Fig. $2 \mathrm{C}$ and these data follow the same pattern as the NSF test data. In fact, a two-way ANOVA revealed that lentiviral-mediated overexpression of BDNF in the hippocampus, but not in the cortex, increased sucrose preference. Indeed, there was significant 

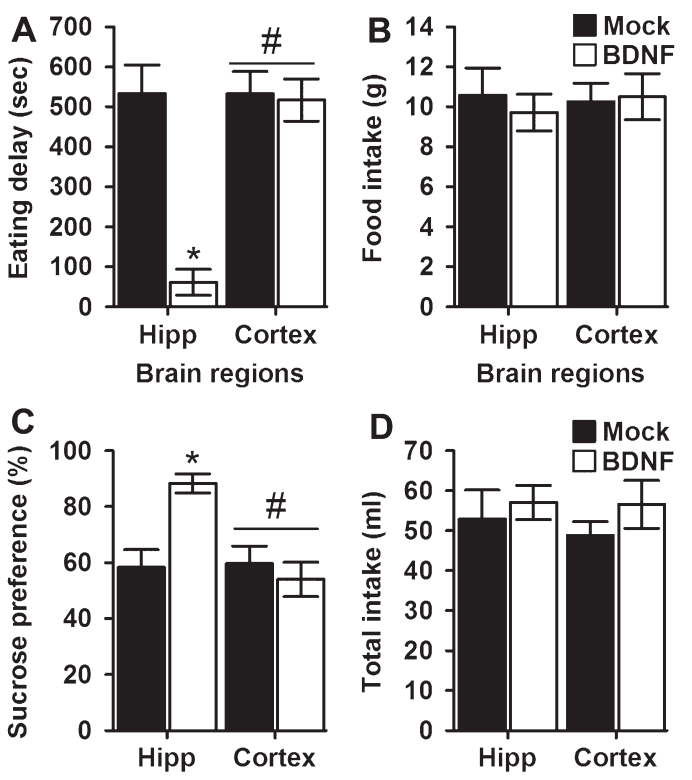

Brain regions

Figure 2 Effect of lentiviral-mediated BDNF overexpression on chronic social defeat-induced depression-like behavior in the novelty suppressed feeding (NSF) and sucrose preference test (SPT). (A) Eating delay as measured by the latency to bite food in a novel anxiogenic environment following $24-\mathrm{h}$ food deprivation, (B) food intake of rats in the home cage during a 90 min period following NSF latency test, (C) sucrose preference was calculated by the following formula: preference $=$ (sucrose solution intake/total fluid intake) $\times 100$, and (D) total fluid intake sucrose intake + water intake. *Significant differences between Mock and BDNF $(p<0.05)$. " Significant differences between the hippocampus and the cortex $(p<0.05)$ (two-way ANOVA, Bonferroni's post hoc test). Hippocampus (LV-Mock $n=9$, LVBDNF $n=10$ ); cortex (LV-Mock $n=8$, LV-BDNF $n=8$ ).

main effect of brain region $\left(F_{(1,31)}=8.897, p=0.006\right)$ and viral injection $\left(F_{(1,31)}=4.856, p=0.035\right)$. More importantly, the interaction between viral-injection and the brain region was significant $\left(F_{(1,31)}=10.433, p=0.003\right)$. As a possibly
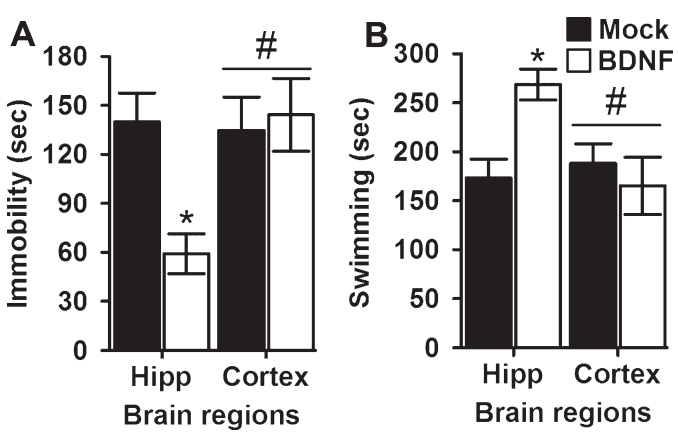

Figure 3 Effect of lentiviral-mediated BDNF overexpression on chronic social defeat-induced depression-like behavior in the forced-swim test (FST). (A) Immobility and (B) swimming. *Significant differences between Mock and BDNF $(p<0.05)$. "Significant differences between the hippocampus and the cortex $(p<0.05)$ (two-way ANOVA, Bonferroni's post hoc test). Hippocampus (LV-Mock $n=9$, LV-BDNF $n=10)$; cortex (LV-Mock $n=8$, LV-BDNF $n=8$ ).

confounding variable for anhedonia, total fluid intake was also tested and results are depicted in Fig. 2D. The two-way ANOVA analysis revealed that there was no main effect of viral injection $\left(F_{(1,31)}=1.170, p=0.288\right)$ nor brain region $\left(F_{(1,31)}=0.169, p=0.863\right)$ on total fluid intake. Interestingly, the interaction between viral-injection and brain region was not significant $\left(F_{(1,31)}=0.104, p=0.749\right)$. The experiments using the non-stressed control groups are shown in Supplementary Fig. 2. The data with non-stress controls and stress animals are a combination of separate experiments, which is far from the optimal design.

After completion of the SPT, rats were tested in the Forced swim test (FST) 48-h later and results are depicted in Fig. 3. The groups of rats used were as follow after removing 3 rats because of diving: hippocampus (LV-Mock $n=8$, LV-BDNF $n=9$ ); cortex (LV-Mock $n=7$, LV-BDNF $n=8)$. Two-way ANOVA analysis revealed that BDNF overexpression in the hippocampus, but not in the cortex, significantly decreased the time of immobility $(\sim 2.5$ fold $)$ [main effect of brain region: $\left(F_{(1,28)}=4.820, p=0.037\right)$, main effect of viral-injection $\left(F_{(1,28)}=3.849, p=0.060\right)$, the main effect of brain region $\times$ virus interaction $\left(F_{(1,28)}=6.147, p=0.019\right)$ ] (Fig. 3A). Consequently and as expected, when injected into the hippocampus, but not into the cortex, BDNF significantly increased the time the rats spent swimming [main effect of brain region: $\left(F_{(1,28)}=4.217, p=0.049\right)$, main effect of viral-injection $\left(F_{(1,28)}=2.847, p=0.103\right)$, the main effect of the interaction between the viral-injection and the brain region $\left(F_{(1,28)}=7.607, p=0.010\right)$ ] (Fig. 3B). The experiments using the non-stressed control groups are shown in Supplementary Fig. 3. The data with non-stress controls and stress animals are a combination of separate experiments, which is far from the optimal design.

Our present data indicate that lentiviral-mediated overexpression of BDNF into the hippocampus, but not into the cortex, induces reduction of immobility and enhancement in the swimming time in the FST in rats following chronic social defeat stress.

\subsection{Effects of miR124a overexpression and silencing on social defeat stress-induced depression-like behavior in rats}

To test the effects of miR124a on social defeat stressinduced depression like behavior in Wistar rats, both gainand loss-of-function approaches were used. In these experiments, LV-siR124a was used to knock down its expression and a lentivirus expressing miR124a (LV-miR124a) was used to up-regulate its expression in the hippocampus or into the cortex. Empty vector was used as a negative control (LV-Mock).

Rats were first tested in the NSF test and results are depicted in Fig. 4. The groups of rats used were as follow: hippocampus (LV-Mock $n=7$, LV-miR124a $n=8$, siR124a $n=7$ ); cortex (LV-Mock $n=6$, LV-miR124a $n=7$, siR124a $n=7)$. Two-way ANOVA analysis has shown that the latency to feed varied as a function of virus $\left(F_{(2,38)}=13.932\right.$, $p<0.001)$ but not brain region $\left(F_{(1,38)}=1.402, p=0.460\right)$. Nevertheless, the interaction between viral-injection and the brain region was significant $\left(F_{(2,38)}=7.969, p=0.001\right)$ (Fig. 4A). Post hoc evaluations revealed that, compared to 

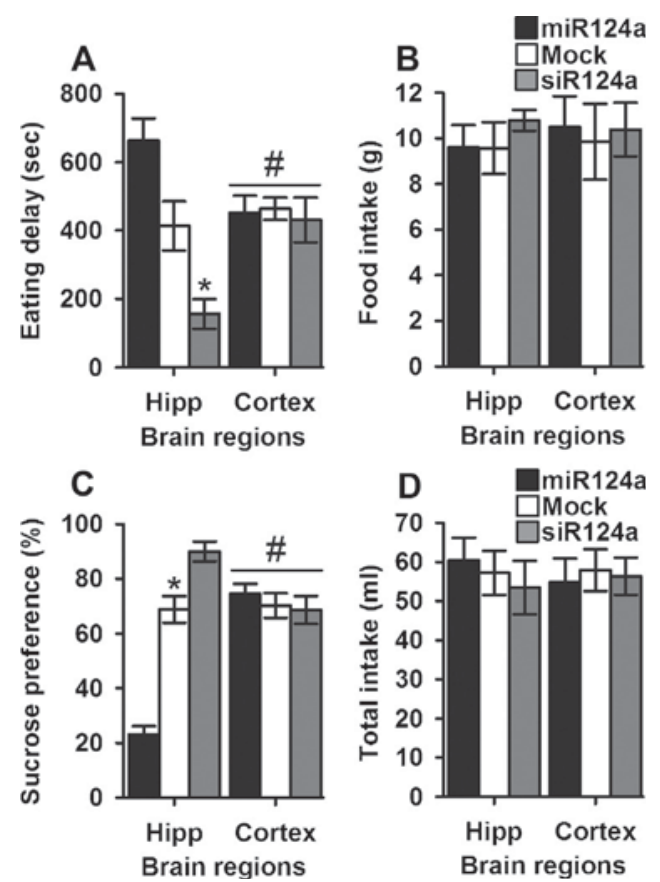

Figure 4 Effect of lentiviral-mediated miR124a modulation on chronic social defeat-induced depression-like behavior in the novelty suppressed feeding (NSF) and sucrose preference test (SPT). (A) Eating delay as measured by the latency to bite food in a novel anxiogenic environment following 24 -h food deprivation, (B) food intake of rats in the home cage during a 90 min period following NSF latency test, (C) sucrose preference was calculated by the following formula: preference $=$ (sucrose solution intake/total fluid intake) $\times 100$. *Significant differences between miR124a or siR124a and Mock $(p<0.05)$. " Significant differences between the hippocampus and the cortex $(p<0.05)$ (two-way ANOVA, Bonferroni's post hoc test), and (D) total fluid intake sucrose intake + water intake. *Significant differences between miR124a or siR124a and Mock ( $p<0.05)$. \#Significant differences between the hippocampus and the cortex $(p<0.05)$ (two-way ANOVA, Bonferroni's post hoc test). Hippocampus (LV-Mock $n=7$, LV-miR124a $n=9$, siR124a $n=8$ ); cortex (LV-Mock $n=6$, LV-miR124a $n=7$, siR124a $n=7$ ).

controls (LV-Mock), rats injected with miR124a showed a ca. 1.5 -fold increased latency to approach the food pellet $(p=0.028)$. More importantly and as expected, silencing miR124a expression into the hippocampus, but not into the cortex, using siR124a drastically reduced the latency to feed $(p=0.034$ and $p<0.001$ vs. Mock and miR124a respectively). In addition and as depicted in Fig. 4B, the threegroups of rats did not differ in their food intake in the home cage during a $90 \mathrm{~min}$ period immediately following the latency test [main effect of brain region: $\left(F_{(1,38)}=0.243\right.$, $p=0.625)$; main effect of viral-injection: $\left(F_{(2,38)}=0.179\right.$, $p=0.837)$; interaction between virus and brain region: $\left.\left(F_{(2,38)}=0.126, p=0.882\right)\right]$. Finally, both miR124a-overexpressing and -silencing groups of rats did not differ in the amount of weight lost during the $24 \mathrm{~h}$ food deprivation period (data not shown). The experiments using the nonstressed control groups are shown in Supplementary Fig. 4. The data with non-stress controls and stress animals are a combination of separate experiments, which is far from the optimal design.

These findings strongly suggest that decreased and increased latency to feed were directly related to lower and higher anxiety levels in siR124a- and miR124a-overexpressing rats respectively.

After completion of the NSF test, the same rats were assed in the sucrose preference test (SPT) using the methodology described in Section 2 above and results are depicted in Fig. $4 C$ and $\mathrm{D}$. The groups of rats used were as follow: hippocampus (LV-Mock $n=7$, LV-miR124a $n=9$, siR124a $n=8$ ); cortex (LVMock $n=6$, LV-miR124a $n=7$, siR124a $n=7$ ). Two-way ANOVA analysis revealed that the average sucrose preference of rats calculated from measures taken in Mock, siRNA124a and miR124a-injected groups differed significantly from each other. In fact and as shown in Fig. 4C, there was a significant main effect of viral-injection $\left(F_{(2,38)}=30.598, p<0.001\right)$ as well as brain region $\left(F_{(1,38)}=9.751, p=0.003\right)$ on sucrose preference. Interestingly, the interaction between the two factor was highly significant $\left(F_{(2,38)}=43.635, p<0.001\right)$. Post hoc evaluations indicated that compared to Mock-, miR124aexpressing rats showed $\sim 3$ folds decreased sucrose preference $(p<0.001)$. In contrast, siR124a-injected rats displayed higher preference for sucrose $(\sim 1.3$ fold) compared to Mock control animals $(p=0.047)$. During the same test, rats were then assessed for their total fluid intake and the two-way ANOVA analysis revealed that no significant differences were observed. As depicted in Fig. 4D, Mock-, siR124a- and miR124ainjected rats consumed the same amount of fluid during the SPT. Indeed, there was no main effect of virus $\left(F_{(2,38)}=0.142\right.$, $p=0.868)$ and no significant main effect of the brain region $\left(F_{(1,38)}=0.018, p=0.894\right)$. More importantly, the virus $\times$ brain region interaction was not significant $\left(F_{(2,38)}=0.284\right.$, $p=0.754)$. The experiments using the non-stressed control groups are shown in Supplementary Fig. 5. The data with non-stress controls and stress animals are a combination of separate experiments, which is far from the optimal design.

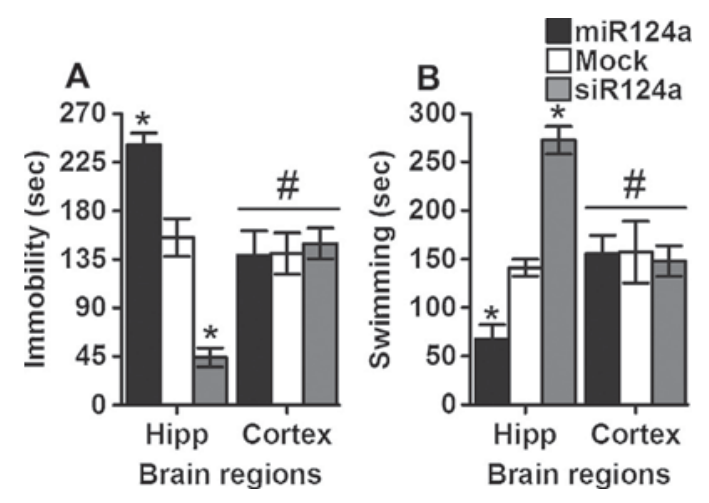

Figure 5 Effect of lentiviral-mediated miR124a modulation on chronic social defeat-induced depression-like behavior in the forced-swim test (FST). (A) Immobility and (B) swimming. *Significant differences between miR124a or siR124a and Mock $(p<0.05)$. " Significant differences between the hippocampus and the cortex $(p<0.05)$ (two-way ANOVA, Bonferroni's post hoc test). Hippocampus (LV-Mock $n=7$, LV-miR124a $n=9$, siR124a $n=8$ ); cortex (LV-Mock $n=6$, LV-miR124a $n=7$, siR124a $n=7)$. 
After completion of the SPT, rats were tested in the FST using these groups of animals: hippocampus (LV-Mock $n=6$, LV-miR124a $n=9$, siR124a $n=8$ ); cortex (LV-Mock $n=6$, LV-miR124a $n=7$, siR124a $n=7$ ). As depicted in Fig. 5, modulation of miR124a expression into the hippocampus profoundly affected FST behavior. In fact, the duration of immobility was affected following viral-injection $\left(F_{(2,38)}=19.285, p<0.001\right)$ but there was no effect of the brain region $\left(F_{(1,38)}=0.087, p=0.770\right)$. Nevertheless, the viral injection $\times$ brain region interaction was highly significant $\left(F_{(2,38)}=24.208, p<0.001\right)$ (Fig. 5A). Post hoc evaluation indicated that miR124a-overexpressing rats were $\sim 1.7$ fold more immobile than Mock control animals ( $p=0.012)$. In contrast and as expected, siR124a-expressing rats were $\sim 3$ folds less immobile than controls $(p=0.005)$. In addition and as shown in Fig. $5 \mathrm{~B}$, the same pattern was also observed when swimming behavior was assessed in these groups. Thus, the two-way ANOVA analysis indicated a significant main effect of viral-injection $\left(F_{(2,38)}=16.586, p<0.001\right)$ and a significant interaction between the brain region and viral-injection $\left(F_{(2,38)}=19.466, p<0.001\right)$. Post hoc evaluations revealed that compared to Mock controls, miR124a spent less time swimming $(p=0.071)$. However, siR124a-expressing rats were more active in their swimming behavior $(p=0.003)$. The experiments using the non-stressed control groups are shown in Supplementary Fig. 6. The data with non-stress controls and stress animals are a combination of separate experiments, which is far from the optimal design.

\section{Discussion}

This is the first study to demonstrate that exposure to chronic social defeat stress can induce miR124a expression in the brain hippocampus, a key brain region associated with depression-like behavior in rats. In addition and as expected, the mRNA expression level of BDNF, which is a direct target of miR124a, was reduced following stress exposure. More importantly, we showed that viral mediated overexpression of miR124a in the hippocampus, but not in the cortex, was pro-depressant. In contrast, BDNF or siR124a overexpression reduced depression-like behavior in rats. These results indicate that miR124a is a critical regulator for BDNF in rat hippocampus and we hypothesize consequently that miR124a might participate in the social defeat stress-induced depressive-like behavior through the regulation of BDNF genetic expression.

Neurotrophic factors such as BDNF were shown to induce changes in hippocampal neurogenesis and mediate the neuropathophysiology of depression (Castren et al., 2007; D'Sa and Duman, 2002; Duman and Monteggia, 2006). To further clarify the neurochemical impact of chronic stress on hippocampal neurotrophic factors expression, we studied mRNA levels of the BDNF after 21-days social defeat stress. Results have shown that, after three weeks, stress induced a pronounced decrease in BDNF expression in the hippocampus but not in the cortex confirming previous reports which found that chronic restraint or social defeat stress caused downregulation of $B D N F$ in the same brain region (Haenisch et al., 2009). In addition, our findings are in line with those of Pizarro and co-workers who found using in situ hybridization, that a brief $(10 \mathrm{~min})$ exposure to intense social stress significantly decreased BDNF mRNA expression in the hippocampus and amygdala $24 \mathrm{~h}$ after social defeat when compared to control (naive) mice (Pizarro et al., 2004). Also, our findings agree with those of Alfonso and colleagues, who had shown that when mice were subjected to repeated restraint stress, hippocampal BDNF messengers were down-regulated as measured by RT-PCR (Alfonso et al., 2006). More importantly, chronic social defeat stress has also been shown to decrease expression of hippocampal BDNF (Komatsu et al., 2011). Finally, it should be notified that the work performed in Nestler lab indicated that detailed mRNA analysis of whole mouse hippocampus revealed that chronic defeat stress induced an approximately threefold reduction in BDNF mRNA levels (Tsankova et al., 2006). Therefore, we could demonstrate that chronic social defeat stress caused brain region specific alterations in the expression of BDNF suggesting that these changes may have implications in brain plasticity and behavioral changes following social stress.

In an attempt to clarify the impact of microRNA on BDNF messengers, we assessed the expression of miR124a in the hippocampus following chronic social defeat stress. MicroRNAs are small non-coding RNAs involved in the regulation of gene expression and protein translation and we hypothesize that miRNAs may be involved in the regulation of chronic stress-related genes in general and depression in particular. Results have shown that reduced BDNF levels were accompanied with an increase of miR124a expression after 21-days social defeat. To the best of our knowledge, we are the first who could demonstrate a negative correlation between levels of BDNF and the expression of miR124a. Indeed, we have shown in previous report that BDNF is a direct target of miR124a (Bahi and Dreyer, 2013; Chandrasekar and Dreyer, 2009). Our findings are in line with those of O'Connor and coworkers who found using microarray and RT-PCR, that earlylife stress affected the expression of multiple hippocampal miRNAs (O'Connor et al., 2013). In fact, using a profiling approach it has been reported that early-life stress affected the expression of multiple hippocampal miRNAs such as miR451 (O'Connor et al., 2013). When investigating the impact of social isolation on genes and associated miRNAs, a recent study reported that the isolated rats exhibited higher levels of miR29 and miR203 (Yang et al., 2013). Importantly, the expression levels of vascular endothelial growth factor A which is a direct target of these miRNAs, was strongly and specifically suppressed (Yang et al., 2013). In our present study we demonstrate for the first time that chronic social defeat increases miR124a expression, suggesting a potential role for depression-associated gene and miRNA interactions via modulation of BDNF expression.

Social stress-induced alterations of gene expression in limbic areas are involved in long-term neuroadaptations and behavioral impairments (Martinez et al., 2002). Interestingly, a number of reports linked BDNF neurotransmission in the neuropathophysiology of psychiatric disorders in general (Balaratnasingam and Janca, 2012) and depression in particular (Duman and Monteggia, 2006). Unfortunately, the molecular mechanism by which BDNF may contribute to the pro-depressant effect of social stress is still not well understood. In the current study we have demonstrated that lentiviral-mediated overexpression of BDNF in the hippocampus, but not into the cortex, results in an anti-depressant like behavior. The current behavioral data are consistent with 
results showing that in male Sprague-Dawley rats, a bilateral infusion of BDNF $(0.25$ and $1.0 \mu \mathrm{g})$ into the dentate gyrus of the hippocampus increases the swimming behavior in the FST and, therefore produces antidepressant-like phenotype (Shirayama et al., 2002). In addition, Govindarajan and coworkers reported that genetically modified mice overexpressing BDNF in excitatory neurons of the forebrain, including the hippocampus, displayed improved performance in the Porsolt FST (Govindarajan et al., 2006). In contrast, heterozygous (+/-) BDNF mice were resistant to the effects of antidepressants in the FST, indicating that antidepressantinduced behavioral effects require regular BDNF signaling (Lindholm et al., 2012; Saarelainen et al., 2003). In addition, it has been shown using the same mice that, when combined with a stress exposure, heterozygous depletion of BDNF resulted in a depressive phenotype in the FST (Duman et al., 2007).

Moreover, we found that hippocampal miR124a overexpression exacerbated stress-induced depression-like behavior. In contrast, inhibition of miR124a signaling in the hippocampus using a lentiviral-mediated silencer expression "rescued" the anti-depressant effect seen in BDNF-overexpressing animals. Therefore, we hypothesize that miR124a plays a pivotal role in stress-related emotions, by regulating the inhibitory effects of social defeat stress on hippocampal BDNF expression. Whatever the underlying mechanisms, our data demonstrate that siR124a and miR124a exert opposite effects on hippocampal BDNF messenger levels, and suggest that the balance between these two factors may play a crucial role in determining vulnerability to stress pro-depressant like effect. Taken together, we propose that social defeat stress exposure increases the expression of miR124a and consequently decreases the expression of BDNF in the hippocampus. Therefore, these results suggest that miR124a silencing has an antidepressant-like effect, at least in part by supporting cell proliferation through the BDNF signaling pathway.

\section{Conclusion}

Our study demonstrates that chronic social defeat impairs depression-like behavior in laboratory animals. The unique expression pattern of gene-miRNA interactions in the hippocampus of socially defeated mice represents a novel aspect of the complex regulatory network involved in emotional illnesses. These results suggest that overexpression of miR124a contributes to chronic social stress induced depression, partially by suppressing BDNF expression. Considering the important role of miR124a in emotions-related brain areas, these results suggest a putative mechanism for chronic social defeat-induced behavioral impairments through the modulation of BDNF. The inhibition of BDNF identified in this study and others (Bahi and Dreyer, 2013; Chandrasekar and Dreyer, 2009) may provide a target for future therapeutics that are directed to tackle psychiatric disorders in general and depression in particular.

\section{Role of the funding source}

$A B$ is receiving funds from the United Arab Emirates University (No. NP/12/04, and NP/13/05) and from the National
Research Foundation (No. 31M082). JLD was supported by Swiss National Science Foundation (Grants nos. 3100-059350, 3100AO-100686, and 31003A-116492). The funders had no role in study design, data collection and analysis, decision to publish, or preparation of the manuscript.

\section{Conflict of interest statement}

The authors report no conflicts of interest.

\section{Acknowledgements}

The authors would like to acknowledge Mrs. Christine Deforel-Poncet for the assistance with viral vectors cloning and preparation. We are grateful also to Mr. Mohamed Elwasila and Mr. Mohamed Shafiullah for their technical assistance.

\section{Appendix A. Supplementary data}

Supplementary material related to this article can be found, in the online version

\section{References}

Acheson, A., Conover, J.C., Fandl, J.P., DeChiara, T.M., Russell, M., Thadani, A., Squinto, S.P., Yancopoulos, G.D., Lindsay, R.M., 1995. A BDNF autocrine loop in adult sensory neurons prevents cell death. Nature 374, 450-453.

Alfonso, J., Frick, L.R., Silberman, D.M., Palumbo, M.L., Genaro, A.M., Frasch, A.C., 2006. Regulation of hippocampal gene expression is conserved in two species subjected to different stressors and antidepressant treatments. Biol. Psychiatry 59, 244-251.

Bahi, A., Boyer, F., Bussard, G., Dreyer, J.L., 2005. Silencing dopamine D3-receptors in the nucleus accumbens shell in vivo induces changes in cocaine-induced hyperlocomotion. Eur. J. Neurosci. 21, 3415-3426.

Bahi, A., Boyer, F., Chandrasekar, V., Dreyer, J.L., 2008a. Role of accumbens BDNF and TrkB in cocaine-induced psychomotor sensitization, conditioned-place preference, and reinstatement in rats. Psychopharmacology 199, 169-182.

Bahi, A., Dreyer, J.L., 2012. Hippocampus-specific deletion of tissue plasminogen activator tPA in adult mice impairs depression- and anxiety-like behaviors. Eur. Neuropsychopharmacol. 22, 672-682.

Bahi, A., Dreyer, J.L., 2013. Striatal modulation of BDNF expression using microRNA124a-expressing lentiviral vectors impairs ethanol-induced conditioned-place preference and voluntary alcohol consumption. Eur. J. Neurosci. 38, 2328-2337.

Bahi, A., Kusnecov, A.W., Dreyer, J.L., 2008b. Effects of urokinasetype plasminogen activator in the acquisition, expression and reinstatement of cocaine-induced conditioned-place preference. Behav. Brain Res. 191, 17-25.

Balaratnasingam, S., Janca, A., 2012. Brain derived neurotrophic factor: a novel neurotrophin involved in psychiatric and neurological disorders. Pharmacol. Ther. 134, 116-124.

Bodnoff, S.R., Suranyi-Cadotte, B., Quirion, R., Meaney, M.J., 1989. A comparison of the effects of diazepam versus several typical and atypical anti-depressant drugs in an animal model of anxiety. Psychopharmacology 97, 277-279.

Cao, X., Pfaff, S.L., Gage, F.H., 2007. A functional study of miR-124 in the developing neural tube. Genes Dev. 21, 531-536.

Castren, E., Rantamaki, T., 2010. The role of BDNF and its receptors in depression and antidepressant drug action: reactivation of developmental plasticity. Dev. Neurobiol. 70, 289-297. 
Castren, E., Voikar, V., Rantamaki, T., 2007. Role of neurotrophic factors in depression. Curr. Opin. Pharmacol. 7, 18-21.

Chandrasekar, V., Dreyer, J.L., 2009. microRNAs miR-124, let-7d and miR-181a regulate cocaine-induced plasticity. Mol. Cell. Neurosci. 42, 350-362.

Chandrasekar, V., Dreyer, J.L., 2011. Regulation of MiR-124, Let-7d, and MiR-181a in the accumbens affects the expression, extinction, and reinstatement of cocaine-induced conditioned place preference. Neuropsychopharmacology 36, 1149-1164.

D'Sa, C., Duman, R.S., 2002. Antidepressants and neuroplasticity. Bipolar Disord. 4, 183-194.

Duman, C.H., Schlesinger, L., Kodama, M., Russell, D.S., Duman, R.S., 2007. A role for MAP kinase signaling in behavioral models of depression and antidepressant treatment. Biol. Psychiatry 61, 661-670.

Duman, R.S., Monteggia, L.M., 2006. A neurotrophic model for stressrelated mood disorders. Biol. Psychiatry 59, 1116-1127.

Govindarajan, A., Rao, B.S., Nair, D., Trinh, M., Mawjee, N., Tonegawa, S., Chattarji, S., 2006. Transgenic brain-derived neurotrophic factor expression causes both anxiogenic and antidepressant effects. Proc. Natl. Acad. Sci. U.S.A. 103, 13208-13213.

Haenisch, B., Bilkei-Gorzo, A., Caron, M.G., Bonisch, H., 2009. Knockout of the norepinephrine transporter and pharmacologically diverse antidepressants prevent behavioral and brain neurotrophin alterations in two chronic stress models of depression. J. Neurochem. 111, 403-416.

Hendrickx, H., McEwen, B.S., Ouderaa, F., 2005. Metabolism, mood and cognition in aging: the importance of lifestyle and dietary intervention. Neurobiol. Aging 26 (Suppl. 1) 1-5.

Hennebelle, M., Balasse, L., Latour, A., Champeil-Potokar, G., Denis, S., Lavialle, M., Gisquet-Verrier, P., Denis, I., Vancassel, S., 2012. Influence of omega-3 fatty acid status on the way rats adapt to chronic restraint stress. PLOS ONE 7, e42142.

Huang, Y.W., Ruiz, C.R., Eyler, E.C., Lin, K., Meffert, M. K., 2012. Dual regulation of miRNA biogenesis generates target specificity in neurotrophin-induced protein synthesis. Cell 148, 933-946.

Karege, F., Bondolfi, G., Gervasoni, N., Schwald, M., Aubry, J.M., Bertschy, G., 2005. Low brain-derived neurotrophic factor (BDNF) levels in serum of depressed patients probably results from lowered platelet BDNF release unrelated to platelet reactivity. Biol. Psychiatry 57, 1068-1072.

Komatsu, H., Ohara, A., Sasaki, K., Abe, H., Hattori, H., Hall, F.S., Uhl, G.R., Sora, I., 2011. Decreased response to social defeat stress in mu-opioid-receptor knockout mice. Pharmacol. Biochem. Behav. 99, 676-682.

Korte, S.M., Smit, J., Bouws, G.A., Koolhaas, J.M., Bohus, B., 1990. Behavioral and neuroendocrine response to psychosocial stress in male rats: the effects of the $5-\mathrm{HT} 1 \mathrm{~A}$ agonist ipsapirone. Horm. Behav. 24, 554-567.

Lindholm, J.S., Autio, H., Vesa, L., Antila, H., Lindemann, L., Hoener, M.C., Skolnick, P., Rantamaki, T., Castren, E., 2012. The antidepressant-like effects of glutamatergic drugs ketamine and AMPA receptor potentiator LY 451646 are preserved in $\operatorname{bdnf}(+) /(-)$ heterozygous null mice. Neuropharmacology 62, 391-397.

Makeyev, E.V., Zhang, J., Carrasco, M.A., Maniatis, T., 2007. The MicroRNA miR-124 promotes neuronal differentiation by triggering brain-specific alternative pre-mRNA splicing. Mol. Cell 27, 435-448.

Martinez, M., Calvo-Torrent, A., Herbert, J., 2002. Mapping brain response to social stress in rodents with c-fos expression: a review. Stress 5, 3-13.

Masi, G., Brovedani, P., 2011. The hippocampus, neurotrophic factors and depression: possible implications for the pharmacotherapy of depression. CNS Drugs 25, 913-931.

Nestler, E.J., Barrot, M., DiLeone, R.J., Eisch, A.J., Gold, S.J., Monteggia, L.M., 2002. Neurobiology of depression. Neuron 34, $13-25$.
O’Connor, R.M., Grenham, S., Dinan, T.G., Cryan, J.F., 2013. microRNAs as novel antidepressant targets: converging effects of ketamine and electroconvulsive shock therapy in the rat hippocampus. Int. J. Neuropsychopharmacol. 16, 1885-1892.

Paxinos, G., Watson, C., 1998. The Rat Brain in Stereotaxic Coordinates. Academic Press, San Diego.

Pizarro, J.M., Lumley, L.A., Medina, W., Robison, C.L., Chang, W.E., Alagappan, A., Bah, M.J., Dawood, M.Y., Shah, J.D., Mark, B., Kendall, N., Smith, M.A., Saviolakis, G.A., Meyerhoff, J.L., 2004. Acute social defeat reduces neurotrophin expression in brain cortical and subcortical areas in mice. Brain Res. 1025, 10-20.

Porsolt, R.D., Bertin, A., Jalfre, M., 1977. Behavioral despair in mice: a primary screening test for antidepressants. Arch. Int. Pharmacodyn. Ther. 229, 327-336.

Qi, L., Hu, Y., Zhan, Y., Wang, J., Wang, B.B., Xia, H.F., Ma, X., 2012. A SNP site in pri-miR-124 changes mature miR-124 expression but no contribution to Alzheimer's disease in a Mongolian population. Neurosci. Lett. 515, 1-6.

Saarelainen, T., Hendolin, P., Lucas, G., Koponen, E., Sairanen, M., MacDonald, E., Agerman, K., Haapasalo, A., Nawa, H., Aloyz, R., Ernfors, P., Castren, E., 2003. Activation of the TrkB neurotrophin receptor is induced by antidepressant drugs and is required for antidepressant-induced behavioral effects. J. Neurosci. 23, 349-357.

Schinder, A.F., Poo, M., 2000. The neurotrophin hypothesis for synaptic plasticity. Trends Neurosci. 23, 639-645.

Schmidt, H.D., Duman, R.S., 2010. Peripheral BDNF produces antidepressant-like effects in cellular and behavioral models. Neuropsychopharmacology 35, 2378-2391.

Schmidt, H.D., Shelton, R.C., Duman, R.S., 2011. Functional biomarkers of depression: diagnosis, treatment, and pathophysiology. Neuropsychopharmacology 36, 2375-2394.

Sen, S., Duman, R., Sanacora, G., 2008. Serum brain-derived neurotrophic factor, depression, and antidepressant medications: meta-analyses and implications. Biol. Psychiatry 64, 527-532.

Shirayama, Y., Chen, A.C., Nakagawa, S., Russell, D.S., Duman, R.S., 2002. Brain-derived neurotrophic factor produces antidepressant effects in behavioral models of depression. J. Neurosci. 22, 3251-3261.

Sofia, R.D., Knobloch, L.C., 1976. Comparative effects of various naturally occurring cannabinoids on food, sucrose and water consumption by rats. Pharmacol. Biochem. Behav. 4, $591-599$.

Soppet, D., Escandon, E., Maragos, J., Middlemas, D.S., Reid, S.W., Blair, J., Burton, L.E., Stanton, B.R., Kaplan, D.R., Hunter, T., Nikolics, K., Parada, L.F., 1991. The neurotrophic factors brainderived neurotrophic factor and neurotrophin-3 are ligands for the trkB tyrosine kinase receptor. Cell 65, 895-903.

Treiber, T., Treiber, N., Meister, G., 2012. Regulation of microRNA biogenesis and function. Thromb. Haemost. 107, 605-610.

Tsankova, N.M., Berton, O., Renthal, W., Kumar, A., Neve, R.L., Nestler, E.J., 2006. Sustained hippocampal chromatin regulation in a mouse model of depression and antidepressant action. Nat. Neurosci. 9, 519-525.

Yamada, K., Nabeshima, T., 2003. Brain-derived neurotrophic factor/TrkB signaling in memory processes. J. Pharmacol. Sci. 91, 267-270.

Yang, L., Engeland, C.G., Cheng, B., 2013. Social isolation impairs oral palatal wound healing in Sprague-Dawley rats: a role for miR-29 and miR-203 via VEGF suppression. PLOS ONE 8, e72359.

Zigova, T., Pencea, V., Wiegand, S.J., Luskin, M.B., 1998. Intraventricular administration of BDNF increases the number of newly generated neurons in the adult olfactory bulb. Mol. Cell. Neurosci. 11, 234-245.

Zurita, A., Murua, S., Molina, V., 1996. An endogenous opiate mechanism seems to be involved in stress-induced anhedonia. Eur. J. Pharmacol. 299, 1-7. 